\title{
Atypische Beschäftigung
}

\author{
Auf der Suche nach den Grenzen zwischen Missbrauch und \\ gewünschter sowie sozialpolitisch vertretbarer Flexibilität
}

\section{Marcel Kirisits}

In den vergangenen zwei Jahrzehnten ist auf dem österreichischen Arbeitsmarkt eine Zunahme von Beschäftigungsverhältnissen festzustellen, die vom so genannten Normalarbeitsverhältnis, das durch abhängige, vollzeitliche, kontinuierliche und auf geregelter Arbeitszeit und geregeltem Einkommen basierende Tätigkeit charakterisiert wird, abweichen. Alle Beschäftigungsverhältnisse, die dieser Norm nicht entsprechen, werden in der Literatur unter dem Sammelbegriff "atypische Beschäftigung" zusammengefasst. Darunter fallen so unterschiedliche Ausprägungen wie Teilzeitarbeit, geringfügige Beschäftigung, befristete Beschäftigung, Leiharbeit, (Schein-)Selbstständigkeit in Form von Werkund freien Dienstverträgen sowie Arbeit auf Abruf und Telearbeit. Aus arbeitsmarkt- und (interessen-)politischer Sicht ist diese Kategorisierung trotz mitunter berechtigter Kritik daran ${ }^{1}$ insofern von Bedeutung, als es sich bei atypischen Beschäftigungsverhältnissen teilweise um Vertragsformen handelt, deren arbeits- und sozialrechtliche Einbettung beträchtliche Mängel aufweist. Abgesehen von diesen Defiziten birgt atypische Beschäftigung die Gefahr in sich, eine Struktur von In- und Outsidern auf dem Arbeitsmarkt zu schaffen oder bestehende Unterschiede zu verstärken. Die Insider im weiteren Sinne befinden sich in einem (relativ) sicheren Vollzeit-Beschäftigungsverhältnis und genießen sämtliche arbeits- und sozialrechtlichen Ansprüche sowie etwaige Sozialleistungen innerhalb ihrer Unternehmen. Zu den Insidern im engeren Sinne könnte man jene Personengruppe zählen, der sich zusätzlich Aufstiegs- bzw. Karrieremöglichkeiten eröffnen. Die Outsider kennzeichnen häufige Jobwechsel, Phasen der Arbeitslosigkeit, Beschäftigungsverhältnisse mit mangelhafter arbeits- und sozialrechtlicher Einbettung, prekäre Einkommenssituation und fehlende berufliche Aufstiegsperspektiven. Dieser Beitrag zeichnet die Entwicklung atypischer Beschäftigungsverhältnisse in der Steiermark nach, beleuchtet die damit verbundenen Probleme bei der Beurteilung atypischer Beschäftigung und widmet sich abschließend 
dem Bereich neuer Selbstständigkeit und den damit verbundenen Abgrenzungsproblemen zur Unselbstständigkeit.

\section{Atypische Beschäftigung in der Steiermark}

Die vorhin skizzierte Entwicklung war und ist auch ein wesentliches Merkmal des steirischen Arbeitsmarktes. In wenigen Worten: starke Zunahme von atypischen Beschäftigungsverhältnissen ${ }^{2}$ mit dem Schwerpunkt auf Teilzeitarbeit und geringfügiger Beschäftigung, wovon großteils Frauen betroffen sind; die Bereiche Leiharbeit, freie Dienstverträge und Werkverträge zeigen, wenngleich in absoluten Zahlen noch unbedeutend, eine beträchtliche Dynamik. Die Ergebnisse für die Steiermark entsprechen somit der Entwicklung im restlichen Bundesgebiet. ${ }^{3}$ Insgesamt stieg die Zahl der unselbstständig Beschäftigten in der Steiermark zwischen 1980 und 2001 von 384.883 auf $423.281 .{ }^{4}$ Dies entspricht einer Steigerung von knapp 10 Prozent für den gesamten Zeitraum von 21 Jahren.

\subsection{Teilzeitarbeit}

Teilzeitbeschäftigung ist vorwiegend Frauenbeschäftigung. Von allen steirischen Teilzeitbeschäftigten waren im Jahr 2001 mehr als 90 Prozent weiblich. Im Zeitraum 1980 bis 2001 stieg die Teilzeitquote bei Frauen von 13,4 auf 31,6 Prozent (absolut von 15.800 auf 55.300), während sie bei Männern nahezu unverändert blieb. ${ }^{5}$ Somit war 2001 fast ein Drittel aller beschäftigten Frauen in Teilzeit. Die für weibliche Teilzeit typischen Branchen sind der Handel, das Gesundheits- und Sozialwesen sowie das Realitätenwesen. Insgesamt vereinigen diese Sektoren etwa 55 Prozent aller teilzeitbeschäftigten Frauen. Sowohl die Männer- als auch die Frauenteilzeitbeschäftigung liegen in diesen Sektoren über dem allgemeinen Durchschnitt. Interessant dabei ist auch die Aufschlüsselung der Erwerbsbeteiligung von Frauen nach Vollzeit- und Teilzeitarbeitsplätzen. So stiegen von 1980 bis 1990 die Vollzeitarbeitsplätze für Frauen in der Steiermark um rund 25 Prozent, während sie von 1990 bis 2001 um etwa sieben Prozent abnahmen. Das heißt, dass die gestiegene Erwerbsbeteiligung von steirischen Frauen in den letzten zehn Jahren im Wesentlichen auf die Zunahme von Teilzeitarbeitsplätzen zurückzuführen ist.

\subsection{Geringfügige Beschäftigung}

Seit 1995 hat sich die Anzahl der geringfügig Beschäftigten ${ }^{6}$ in der Steiermark um 30 Prozent auf 31.357 im Jahr 2001 erhöht. Ähnlich der Teilzeitbeschäftigung wird auch geringfügige Beschäftigung vorwiegend von Frauen ausgeübt. So waren 2001 mehr als 70 Prozent aller gering- 
fügig Beschäftigten weiblich. Branchenbezogen entfällt der größte Anteil bei den Frauen auf den Einzelhandel, gefolgt von unternehmensbezogenen Dienstleistungen, ${ }^{7}$ Beherbergungs- und Gaststättenwesen sowie Gesundheits-, Veterinär- und Sozialwesen.

Eine statistische Sonderauswertung auf Bundesebene ergab, dass mehr als die Hälfte aller geringfügig beschäftigten Frauen ausschließlich in einem geringfügigen Beschäftigungsverhältnis steht, während dies auf nur etwas mehr als ein Drittel der Männer zutrifft. Mehr als sechzig Prozent aller geringfügig beschäftigten Männer haben somit ein zusätzliches Versicherungsverhältnis über eine andere Erwerbstätigkeit, eine Eigenpension bzw. über die Arbeitslosen- und Krankenversicherung, während dies nur für etwa 40 Prozent der geringfügig beschäftigten Frauen gilt. ${ }^{8}$

\subsection{Befristete Arbeitsverhältnisse}

Daten über befristete Arbeitsverhältnisse ${ }^{9}$ liegen, mit Ausnahme der Leiharbeit, nur auf Bundesebene vor. Seit der ersten Erhebung befristeter Erwerbstätigkeit im Jahre 1995 ist der Anteil befristet Beschäftigter an den unselbstständig Erwerbstätigen nur unwesentlich gestiegen. Der Anstieg von 3,7 Prozent im Jahr 1995 auf 4,3 Prozent im Jahr 2001 lässt auf keine starke Dynamik in diesem Beschäftigungssegment schließen, zumal der Anteil 1997 bereits bei 4,3 Prozent lag. Allerdings sind auch befristete Arbeitsverhältnisse geschlechtsspezifisch ungleich verteilt. So gab es im März 2001 90.000 Frauen, aber nur 46.000 Männer mit Befristungen, was einer Befristungsquote von 6,3 Prozent bei Frauen und 2,6 Prozent bei Männern entspricht. ${ }^{10}$ Diese Zahlen enthalten allerdings keine Ausbildungsverträge, die immerhin rund 50 Prozent aller Befristungen ausmachen.

\subsection{Leiharbeit}

Nach einer relativ geringen Steigerung Anfang der neunziger Jahre nimmt die Anzahl der überlassenen Arbeitskräfte in der Steiermark seit 1994 kontinuierlich zu. Im Jahr 2001 befanden sich mehr als 5.000 Personen in Leiharbeit, während es 1990 erst 1.400 waren. ${ }^{11}$ Im Zeitraum 1990 bis 2001 hat sich sowohl die Anzahl der Überlasserbetriebe als auch jene der Beschäftigerbetriebe vervierfacht. Hinsichtlich der Branchenverteilung ist der industrielle Sektor mit einem Anteil von etwa 50 Prozent an der gesamten Leiharbeit dominant. Der Anteil der Arbeiterlnnen an den gesamten Leiharbeiterlnnen bewegt sich in den hinsichtlich Leiharbeit dominanten Branchen (Industrie, Gewerbe und Handwerk) in einer Bandbreite von 80 bis 90 Prozent. 


\subsection{Freie Dienstverträge - Werkverträge}

Grundsätzlich ist zu unterscheiden zwischen freiem Dienstvertrag, Werkvertrag mit Gewerbeschein und Werkvertrag ohne Gewerbeschein. Die Werkvertragsnehmer ohne Gewerbeschein werden gemeinhin auch als "neue Selbstständige“ bezeichnet. Der Werkvertrag gilt als Zielschuldverhältnis (ein Werk wird erbracht), während ein freier Dienstvertrag den Dauerschuldverhältnissen zuzuordnen ist. Statistische Daten über freie Dienstverträge und Werkverträge liegen erst seit 1998 vor. Die Daten sind, weil sie eben erst für einen relativ kurzen Zeitraum zur Verfügung stehen, mit der gebotenen Vorsicht zu interpretieren. In Relation zur gesamten Erwerbstätigkeit hält sich die Verbreitung von freien Dienstverträgen ${ }^{12}$ und Werkverträgen (noch) in Grenzen, wenngleich diese Bereiche in den vergangenen Jahren eine starke Dynamik erfahren haben. Die freien Dienstverträge in der Steiermark haben von 1998 bis 2001 um 50 Prozent (absolut von 2.018 auf 3.017) zugenommen, wobei freie Dienstverträge fast zu 90 Prozent im Angestelltenbereich zu finden sind und sich etwas stärker auf Männer (53 Prozent) als auf Frauen verteilen. Im Bereich der neuen Selbstständigkeit (Werkvertragsnehmer ohne Gewerbeschein) ist es zu einer ähnlichen Entwicklung wie bei den freien Dienstverträgen gekommen. Absolut betrachtet stieg die Anzahl der neuen Selbstständigen in der Steiermark von 565 im Jahresdurchschnitt 1998 auf 2.536 im Jahr 2001. ${ }^{13}$

\section{Das Problem der Beurteilung atypischer Beschäftigung}

Grundsätzlich lassen sich verschiedene Merkmale für die Charakterisierung von Beschäftigungsverhältnissen anführen, die die Weisungsgebundenheit des Arbeitnehmers, die Entlohnungsform und andere beschäftigungsrelevante Aspekte betreffen (Tabelle 1). Die Ausprägungen in der Realität bewegen sich innerhalb der in der Tabelle aufgelisteten Extrempole. Um es beispielhaft zu verdeutlichen: Auf der linken Seite der Tabelle finden sich die Merkmale eines pragmatisierten Beamtentums mit vollständiger arbeits- und sozialrechtlicher Einbettung, auf der rechten Seite die eines Werkvertragsnehmers mit kurzen Zeithorizonten, großen Unsicherheiten, keinen arbeitsrechtlichen und geringen sozialrechtlichen Ansprüchen. ${ }^{14}$ Theoretisch sind - je nach Ausgestaltung mannigfaltigste Formen der Beschäftigung denkbar, de facto wird der Handlungsspielraum der Akteure (ArbeitgeberInnen und Arbeitnehmerlnnen) aber durch die Rechtsordnung und den institutionellen Rahmen definiert und begrenzt. Diese Beschränkungen gelten mehr oder weniger auch für atypische Beschäftigungsformen. 


\section{Tabelle 1: Strukturmerkmale von Erwerbstätigkeit}

\begin{tabular}{|c|c|c|}
\hline \multicolumn{3}{|c|}{ Weisungsgebundenheit } \\
\hline $\begin{array}{c}\text { gegenüber dem Arbeitgeber } \\
\Downarrow \\
\text { abhängige Beschäftigung }\end{array}$ & $\Leftrightarrow$ & $\begin{array}{c}\text { Kundenorientierung } \\
\Downarrow \\
\text { selbst. Tätigkeit }\end{array}$ \\
\hline \multicolumn{3}{|c|}{ Entlohnung } \\
\hline erfolgsunabhängig & $\Leftrightarrow$ & erfolgsabhängig \\
\hline \multicolumn{3}{|c|}{ Dauer und Lage der Arbeitszeit } \\
\hline $\begin{array}{c}\text { Regelarbeitszeit } \\
\text { Vollzeit } \\
\text { Betriebszeit = Arbeitszeit }\end{array}$ & & $\begin{array}{l}\text { flexible Jahresarbeitszeit } \\
\text { geringfügige Beschäftigung } \\
\text { Betriebszeit } \neq \text { Arbeitszeit }\end{array}$ \\
\hline \multicolumn{3}{|c|}{ Beschäftigungssicherheit } \\
\hline Unkündbarkeit & $\Leftrightarrow$ & Vertragsfreiheit \\
\hline \multicolumn{3}{|c|}{ Soziale Sicherheit } \\
\hline Sozialversicherungspflicht & $\Leftrightarrow$ & Sozialversicherungsfreiheit \\
\hline
\end{tabular}

Quelle: Hoffmann, Walwei (1998) 411.

Zur Verfeinerung der Beurteilungsinstrumente für Beschäftigungsverhältnisse bieten sich Indikatoren an, die Hinweise liefern sollen, inwieweit ein Beschäftigungsverhältnis bestimmte Risiken in sich birgt und somit prekären Charakter bekommt. Tabelle 2 listet einige der dafür vorgeschlagenen Kriterien auf. 


\section{Tabelle 2: Merkmale prekärer Beschäftigungsverhältnisse}

- Kurzer Zeithorizont

- Hohes Risiko des Job-/Auftragsverlustes

- Wenige Kontrollmöglichkeiten der Beschäftigten hinsichtlich Arbeitsbedingungen, Löhnen etc.

- Gesetze (und/oder Kollektivvertrag) bieten wenig arbeitsrechtlichen und sozialen Schutz

- Niedrige Einkommen, verknüpft mit Verarmung und schlechter sozialer Einbeziehung

Die Merkmale in Tabelle 2 geben eine Orientierung für die Beurteilung und Einordnung atypischer Vertragsarten. Sie treffen in unterschiedlichem Ausmaß auf bestimmte Formen atypischer Beschäftigung zu, sind aber durchaus nicht auf diese beschränkt. Weiters ist zu berücksichtigen, dass die unterschiedlichen Abstufungen von Prekarität nur eingebettet in den biografischen Zusammenhang einer Person hinreichend erfasst werden können: Sowohl persönliche Merkmale wie Ausbildung, familiärer Status und ethnischer Hintergrund als auch Zukunftsperspektiven der Betroffenen müssen in die Beurteilung einfließen. Nicht die einzelnen Dimensionen, sondern die Kombination der Faktoren machen ein prekäres Beschäftigungsverhältnis aus. ${ }^{15}$

Auf Basis der in Tabelle 2 aufgelisteten Merkmale dürften Werkverträge den höchsten Grad an Prekarisierung aufweisen, während Teilzeitarbeit und Leiharbeit (mit Inkrafttreten des Kollektivvertrages am 1. März 2002) zumindest bezüglich ihrer arbeits- und sozialrechtlichen Einbettung vergleichsweise unproblematisch sind. Allerdings treten bei zu geringer Stundenanzahl im Teilzeitbereich für die (vorwiegend weiblichen) Betroffenen erhebliche finanzielle Schwierigkeiten auf, während der kurze Zeithorizont ein Manko von Leiharbeit ${ }^{16}$ und befristeten Arbeitsverhältnissen darstellt. Was die Einkommenssituation atypisch Beschäftigter betrifft, kommen Fink, Riesenfelder und Tálos (2001) auf ein monatliches Gesamteinkommen (inkl. anderer Einkommen der Person) von unter 5.000 Schilling (363 Euro) für zwei Drittel aller geringfügig Beschäftigten. Für geringfügig Beschäftigte konstatieren dieselben Autoren ein bedeutend höheres Armutsrisiko als im österreichischen Gesamtschnitt ${ }^{17}$, für freie Dienstnehmerlnnen ein überdurchschnittliches Risiko. ${ }^{18}$ Auch wenn die "Prekarisierungsgrade" je nach atypischer Beschäftigungsform und Lebenssituation differieren, lassen die bisher vorliegenden Studien und Statistiken den Schluss zu, dass es zu einem schrittweisen Anstieg prekärer Beschäftigung auf dem österreichischen Arbeitsmarkt gekommen ist. 


\section{Warum sind Atypische atypisch?}

Letztlich könnte man die im Zusammenhang mit atypischer Beschäftigung entstehenden Probleme mit dem Hinweis vom Tisch wischen, dass es jedem/jeder freisteht, derartige Beschäftigungsverhältnisse einzugehen oder nicht. Wenn dem so ist und die Freiwilligkeit der Betroffenen im Vordergrund steht, kann man die Absicht, an der bestehenden Situation etwas zu ändern, mit dem Etikett der Zwangsbeglückung versehen und alle Lösungs- bzw. Veränderungsvorschläge als überflüssig abtun. ${ }^{19}$ Für den Umgang mit atypischer Beschäftigung ist es daher wichtig, die Motive der betroffenen ArbeitnehmerInnen zu erfragen und hinterfragen, um darauf aufbauend etwaige Handlungsanleitungen auf politischer Ebene ableiten zu können.

Hinsichtlich der von Frauen dominierten Teilzeitarbeit fällt auf, dass für sechs von zehn Frauen familiäre Gründe für die Wahl von Teilzeit ausschlaggebend waren, während etwa ein Viertel der in Teilzeit arbeitenden Männer keine Vollzeitarbeit finden konnte. Insgesamt üben rund 15 Prozent aller teilzeitbeschäftigten Frauen und 25 Prozent aller teilzeitbeschäftigten Männer ihre Arbeit in dieser Form unfreiwillig ${ }^{20}$ aus (Tabelle 3).

\section{Tabelle 3: Gründe für Teilzeit (Angaben in Prozent)}

\begin{tabular}{|lrr|}
\hline Grund & Männer & Frauen \\
\hline Familiäre Gründe & 14,4 & 58,8 \\
\hline Keine Vollzeittätigkeit gefunden & 25,5 & 14,6 \\
\hline Keine Vollzeittätigkeit erwünscht & 15,7 & 13,7 \\
\hline Schul- oder Berufsaus-I-fortbildung & 20,3 & 3,0 \\
\hline Sonstiges & 20,4 & 8,7 \\
\hline Krankheit & 3,7 & 1,2 \\
\hline & 100,0 & 100,0 \\
\hline
\end{tabular}

Quelle: Arbeitskräfteerhebung 1998 in: Statistische Nachrichten 11 (1999) 945.

Obwohl nur rund 15 Prozent der Frauen laut Befragung unfreiwillig in Teilzeit arbeiten, liegt der tatsächliche Anteil vermutlich höher. Wenn 60 Prozent der Frauen familiäre Gründe für die Wahl von Teilzeit nennen, so dürfte dahinter ein hohes Potenzial an Unfreiwilligkeit stecken. Das Vereinbarkeitsgebot hinsichtlich Beruf und Familie sowie die Tatsache, dass viele Frauen aufgrund fehlender Kinderbetreuungseinrichtungen keine Vollzeitarbeit annehmen können, relativieren das Ausmaß an Freiwilligkeit. 
Ebenfalls weiblich dominiert, aber bezüglich der Motivlagen anders strukturiert zeigt sich die geringfügige Beschäftigung. Demnach greift mehr als ein Drittel der geringfügig Beschäftigten diese Beschäftigungsform mit der Intention des Nebeneinkommens in einer Ausbildungsphase auf. 15 Prozent nutzen die Möglichkeit dieses Einkommenserwerbs neben der Kinderbetreuung, während für 13 Prozent geringfügige Beschäftigung die Funktion eines Zusatzeinkommens neben einer regulären unselbstständigen Beschäftigung erfüllt. ${ }^{21} \mathrm{Da}$ geringfügige Beschäftigung über eine vergleichsweise niedrige Einkommensgrenze definiert wird, verwundert es nicht, dass diese Form als Hauptbeschäftigung ungeeignet ist und immer nur als Anhängsel anderer Einkommensquellen (reguläre Beschäftigung, Partner, Eltern, andere Versicherungsverhältnisse) dienen kann. Speziell diese Form atypischer Beschäftigung muss man sich mittel- und langfristig leisten können. Dieser Überlegung folgend, kommt ein weiteres Teilergebnis der Studie von Riesenfelder et al. nicht überraschend. Bei den 57 Prozent der befragten geringfügig Beschäftigten, die keinen Wunsch nach einer alternativen Beschäftigungsform äußerten, handelte es sich um Personen mit einem zusätzlichen regulären Dienstverhältnis sowie um SchülerInnen, StudentInnen und PensionistInnen. Die restlichen 43 Prozent gaben an, nach Alternativen in Form einer regulären Vollzeitbeschäftigung (15 Prozent), einer regulären Teilzeitbeschäftigung (18 Prozent) oder einer selbstständigen Tätigkeit (5 Prozent) zu suchen.

Den Hauptteil einer weiteren Form atypischer Beschäftigung, nämlich befristete Arbeitsverträge, bilden Ausbildungsverträge (Lehre), die allerdings im Rahmen dieses Beitrags insofern nicht interessieren, als sie Teil eines gewünschten und auch bewährten dualen Ausbildungssystems sind. Auf den Rest entfallen vorwiegend Probearbeitsverhältnisse (22 Prozent) sowie Personen, die keine Dauerstelle gefunden haben (12 Prozent) oder keine Dauerstelle wünschen (11 Prozent). ${ }^{22}$

Abgesehen vom Abschluss von befristeten Verträgen zwecks Ausbildung wird befristete Beschäftigung von Unternehmen hauptsächlich eingesetzt, um sich von der Eignung eines Arbeitnehmers für die ihm zugedachte Position zu überzeugen. Aus arbeitsrechtlicher Sicht ist dabei das Dienstverhältnis auf Probe von jenem zur Probe zu unterscheiden. Das Dienstverhältnis auf Probe bezeichnet den durch besonders leichte Lösbarkeit charakterisierten Teil eines regelmäßig zeitlich darüber hinausgehenden Dienstverhältnisses, während es sich beim Dienstverhältnis zur Probe um ein Dienstverhältnis auf bestimmte Dauer handelt. Der Zweck beider Dienstverhältnisse liegt in der Erprobung des Dienstnehmers, hinsichtlich der Lösungsmöglichkeiten der Dienstverhältnisse gibt es allerdings Unterschiede. ${ }^{23}$ 
Die individuell-ökonomischen Motive für befristete Beschäftigung überschneiden sich teilweise mit jenen für Leiharbeit. Für den Arbeitnehmer kann die Befristung die Chance auf ein unbefristetes Normalarbeitsverhältnis oder eine temporäre Unterbrechung der Arbeitslosigkeit darstellen. ${ }^{24}$ Erworbene Qualifikationen können angewandt werden, und der Kontakt zur Berufswelt geht nicht verloren. Tabelle 4 listet Gründe auf, warum Leiharbeit trotz der damit verbundenen hohen Mobilitäts- und Flexibilitätsansprüche angenommen wird. ${ }^{25}$

\section{Tabelle 4: Motive für Leiharbeit}

Leiharbeit hat Brückenfunktion zwischen Arbeitslosigkeit und kontinuierlicher Beschäftigung

Finanzielle Vorteile der Leiharbeit (höhere Diäten, Kilometergeld etc.)

Geringe Chance auf „normales“ Dienstverhältnis, Leiharbeit einzige Alternative zur Arbeitslosigkeit (gilt für Problemgruppen des Arbeitsmarktes, z. B. ältere Personen)

Hoch qualifizierte, gut bezahlte Tätigkeit im Bereich des Personal leasing. Teilbereiche des Unternehmens werden ausgelagert („Outsourcing“)

Quelle: nach Juffinger (2000) 20-22.

In der Gruppe der freien Dienstnehmer steht bei mehr als einem Drittel (36 Prozent) mit der Aufnahme eines freien Dienstverhältnisses der Wunsch nach einem Wechsel in einen anderen Beschäftigungstyp im Vordergrund. Für mehr als ein Viertel erfüllt der freie Dienstvertrag die Funktion des Erwerbs neben dem Studium, während nur für acht Prozent der Befragten die Beschäftigungsform des freien Dienstverhältnisses eine längerfristige und vor anderen Einkommensquellen vorrangige Möglichkeit der Beteiligung am Erwerbsleben darstellt. Insgesamt scheinen freie Dienstverträge somit für die Betroffenen eher den Charakter einer Übergangslösung als den einer Langzeitlösung zu haben. Ähnlich differenziert stellt sich die Interessenlage von neuen Selbstständigen dar. Mosberger und Steiner (2002) konstruieren auf Basis von qualitativen Interviews mit Betroffenen zwei große Motivkategorien. In die erste Kategorie fallen demnach neue Selbstständige, die aus einer Ökonomie der Not heraus zum Handeln gezwungen werden, weil sie weder realistische noch akzeptable Alternativen auf dem Arbeitsmarkt vorfinden. Die andere Gruppe umfasst jene Personen, die sich von einer selbstständigen, nicht weisungsgebundenen Tätigkeit angezogen fühlen und autonom und eigenverantwortlich arbeiten wollen. ${ }^{26}$ 
Zweifellos ist es falsch, von vornherein von einem Interessengegensatz zwischen Auftraggeber und Auftragnehmer auszugehen. Man muss allerdings auch nicht gleich so weit wie Reindl (2000) gehen, der die neuen Selbstständigen als Vorreiter eines durch und durch egoistischen und materialistischen Habitus sieht, denen der Gedanke an gesellschaftliche Solidarität, daran, dass die Gesunden für die Kranken, die Starken für die Schwachen, die Jungen für die Alten in die Pflicht genommen werden, fremd ist. Neue Selbstständige seien demnach nur noch bereit zu geben, wenn der "return on investment" für sie hoch genug ist, der Schritt in die neue Selbstständigkeit habe somit durchaus freiwilligen Charakter. ${ }^{27}$ Diese sehr pointierte Einschätzung mag auf einen Teil der neuen Selbstständigen zutreffen, wird der Heterogenität der Motivlagen und Personengruppen aber nicht gerecht.

Zusammenfassend kann festgehalten werden, dass die Motive für die Annahme von atypischen Beschäftigungsformen sehr unterschiedlich sind. Das Argument, ein Großteil der atypisch Beschäftigten sei mit seiner Situation zufrieden, hält einer empirischen Überprüfung jedenfalls nicht stand. Zweifellos entsprechen bestimmte Formen atypischer Beschäftigung auch den Flexibilitätswünschen der Menschen in bestimmten Lebenssituationen. Hinter einer vordergründigen Freiwilligkeit stecken jedoch sehr oft strukturelle Gegebenheiten (fehlende Kinderbetreuung, zu geringes Angebot an Vollzeitarbeitsplätzen), denen sich Arbeit suchende Personen anpassen und scheinbar freiwillig auf atypische Beschäftigung zurückgreifen.

\section{Die fließenden Grenzen zwischen Unselbstständigkeit und Selbstständigkeit}

Ein zentraler Problembereich im Zusammenhang mit Teilbereichen atypischer Beschäftigung ist das Verschwimmen der Grenzen zwischen Unselbstständigkeit und Selbstständigkeit. Das ist insofern problematisch, als die im österreichischen Arbeitsrecht verankerten Ansprüche und Schutzbestimmungen nur (unselbstständigen) Arbeitnehmerlnnen zustehen. Das Arbeitsrecht rückt dabei die persönliche Abhängigkeit in den Mittelpunkt der Beurteilung, ob ein Arbeitsverhältnis überhaupt vorliegt bzw. ob eine Person als Arbeitnehmer gilt. Zu den Kriterien persönlicher Abhängigkeit zählen die Einordnung in den betrieblichen Ordnungsbereich (wie zum Beispiel die Bindung der Beschäftigten an Arbeitszeit, Arbeitsort und Arbeitsabfolge) sowie die Weisungsgebundenheit des Arbeitnehmers, die auf die Verfügung des Dienstgebers über die Arbeitskraft des Dienstnehmers Bezug nimmt. ${ }^{28}$ Freien Dienstverträgen und Werkverträgen fehlt dieses zentrale Merkmal. Das österreichische Arbeitsrecht erfasst damit bestimmte Gruppen von Beschäftigten 
nicht mehr, die zwar de facto unselbstständige, wirtschaftlich abhängige Arbeitnehmer sind, aber nicht mehr oder nur unzureichend vom Arbeitnehmerbegriff erfasst werden. Konkret bedeutet dies für die Betroffenen den Verlust der Ansprüche auf Urlaub, Entgeltfortzahlung im Krankheitsfall, Pflegefreistellung und Sonderzahlungen (13. und 14. Gehalt). Daraus resultiert eine Widersprüchlichkeit, die seit Jahrzehnten alle Diskussionen um das Arbeitsrecht belastet. Obwohl die Frage der wirtschaftlichen Abhängigkeit für die Zuordnung einer Person zur Gruppe der Arbeitnehmer keine Rolle spielt, wird der Inhalt des Arbeitsrechts in erster Linie damit legitimiert, dass es sich bei den ArbeitnehmerInnen um wirtschaftlich Schwache ${ }^{29}$ handelt, die eben wegen dieser Schwäche besonderen Schutz benötigen. Die Entwicklung der letzten Jahre zeigt allerdings, dass es in zunehmendem Maße Personen gibt, die vertragsgemäß Arbeiten für andere verrichten und wirtschaftlich schwach sind, aber aufgrund der Tatsache, dass die vorhin genannten Kriterien der persönlichen Abhängigkeit nicht auf sie zutreffen, nicht als (schutzwürdige) Arbeitnehmerlnnen gelten. ${ }^{30}$

Kritisch zu hinterfragen ist in diesem Zusammenhang auch der viel zitierte Gründerboom in Österreich angesichts einer klar erkennbaren Tendenz zu Selbstbeschäftigungsgründungen, also Unternehmensgründungen ohne eine/n Mitarbeiter/in. Grewer und Reindl (2000) kommen in ihrer Studie über Scheinselbstständigkeit im deutschen Bundesland Saarland zum Ergebnis, dass die Gründungswelle in den 90er Jahren einige Spezifika aufweist, die sie von anderen Gründerzeiten abhebt. Diese neue Gründungswelle habe nämlich eine Form der Selbstständigkeit gebracht, die nicht mehr mit der klassischen Ausprägung einer selbstständigen unternehmerischen oder freiberuflichen Existenz vergleichbar sei. Der Existenzgründer der 90er ist der Tendenz nach ein Einzelkämpfer, und er bleibt in aller Regel allein. Sein Gründungsakt ist immer weniger der Startschuss für die Eröffnung eines Unternehmens und die Einstellung anderer Personen, sondern die Selbstanstellung. Die steigende Zahl von Ein-Mann-Unternehmen sei Ausdruck einer strukturell neuartigen Gründerwelle, die ökonomische und kulturelle Muster, die gemeinhin mit Selbstständigkeit in Verbindung gebracht werden, ins Wanken gebracht habe. Unter den neuen Freiberuflern existiere vielfach eine Form selbstgenügsamer Selbstständigkeit; diese gründeten eigentlich keinen Betrieb, sondern besorgten sich selbst Arbeit. ${ }^{31}$ Der Zuwachs der selbstständigen Erwerbsarbeit ist somit kein Zeichen eines ökonomischen „take-off", sondern Ausdruck eines quantitativen und qualitativen Mangels. Wer sich selbst Arbeit in Form des Selbstunternehmertums beschafft, findet entweder keine Anstellung, oder er kann mit dem existierenden Arbeitsangebot wenig anfangen. Beide Faktoren spielen eine Rolle bei der Ausbreitung des Single-Unternehmertums. ${ }^{32}$ 
Für die Steiermark weist die Statistik der Wirtschaftskammer für den Zeitraum 1993 bis 2001 insgesamt 23.088 neu gegründete Unternehmen aus. Hinsichtlich der Rechtsform entfällt der überwiegende Teil der Neugründungen auf Einzelunternehmen, ${ }^{33}$ die im Durchschnitt des Jahres 2001 einen Anteil von 85,1 Prozent ausmachten. Am zweithäufigsten ist die Rechtsform GesmbH (8,9 Prozent), gefolgt von OEG und KEG, auf die zusammen ein Anteil von 6,2 Prozent entfällt. Der relative Anteil der Einzelfirmen an der Gesamtgründungszahl hat sich in den vergangenen Jahren merklich erhöht, im Jahr 1993 lag er erst bei 73,5 Prozent (Tabelle 5). Die stark intensivierte Gründungstätigkeit wird somit in erster Linie von nicht im Firmenbuch registrierten Unternehmen getragen. ${ }^{34}$

\section{Tabelle 5: Unternehmensneugründungen in der Steiermark}

\begin{tabular}{|c|c|c|}
\hline Jahr & Anzahl & $\begin{array}{c}\text { Anteil der Einzelunternehmen } \\
\text { an den Neugründungen } \\
\text { (in \%) }\end{array}$ \\
\hline 1993 & 1.855 & 73,5 \\
1994 & 1.771 & 72,6 \\
1995 & 1.724 & 73,3 \\
1996 & 2.467 & 77,5 \\
1997 & 2.763 & 78,5 \\
1998 & 2.561 & 79,3 \\
1999 & 2.805 & 82,1 \\
2000 & 3.286 & 82,8 \\
2001 & 3.856 & 85,1 \\
\hline
\end{tabular}

Quellen: Hauth (1999), Schmölzer/Karner (2001), Homepage der WK (www.wko.at).

Einen Überblick über die Struktur von Unternehmensgründungen in der Steiermark gibt die Studie von Kirchengast, Mayrhofer und Penker (1999). Ein Teilergebnis dieser Studie war, dass rund die Hälfte der steirischen Jungunternehmer ohne eine/n Beschäftigte/n beginnt, also der Anteil von so genannten Selbstbeschäftigungsgründungen 50 Prozent beträgt. Die Autoren zitieren in diesem Zusammenhang eine Untersuchung von Wanzenböck (1998), die in einer Untersuchung zum selben Thema für Österreich im Gesamten eine deutliche Verschiebung von Mitarbeitergründungen hin zu Selbstbeschäftigungsgründungen seit 1990 konstatiert. Unter anderem gelangt Wanzenböck zu folgendem Schluss: „Ein oft beobachtetes Phänomen, insbesondere bei den Selbstbeschäftigungsgründungen, ist die enge Kooperation der Gründer mit ihren früheren Dienstgebern. Vielfach wird die gleiche Leistung, die früher im Rahmen eines Dienstverhältnisses ausgeübt wurde, nunmehr im 
Rahmen eines Werkvertrages erbracht. Diese Vorgangsweise reduziert für den Auftraggeber bzw. ehemaligen Dienstgeber das Risiko von Leerkosten und ermöglicht dem Auftragnehmer, Aufträge von anderen Auftraggebern anzunehmen. ${ }^{\text {“35 }}$ Ein weiteres Indiz für das "Hineinrutschen“ von ArbeitnehmerInnen in die Selbstständigkeit könnte ein Ergebnis derselben Autorin im Rahmen einer anderen Untersuchung sein. Demnach scheitern „Selbstbeschäftigungsunternehmer" weit weniger häufig, als es ihrem Anteil an den Unternehmensgründungen entsprechen würde. Diese Tatsache kann durchaus dahingehend interpretiert werden, dass das Unternehmerrisiko der "Selbstbeschäftigungsunternehmer" ein geringeres ist, eben weil sie in vielen Fällen nicht wirklich selbstständig sind, sondern nur der Form nach (also Scheinselbstständige), und das Risiko des Scheiterns weiterhin beim Auftraggeber (Arbeitgeber) liegt. Zu vermuten ist, dass - ähnlich der Entwicklung in Deutschland - ein Teil des Gründerbooms darauf basiert, dass Mitarbeiter nun als „Selbstständige" (Werkvertragsnehmer mit Gewerbeschein) Arbeiten verrichten, die sie vorher als unselbstständige Voll- oder Teilzeitbeschäftigte zu erledigen hatten. Diese Vertragsänderung bedeutet für die Betroffenen allerdings den Verlust wesentlicher arbeitsrechtlicher Ansprüche und die Übernahme von Risiken, die sich als Folge von Auftragsschwankungen ergeben.

\section{Vom Arbeitnehmer zum Arbeitskraftunternehmer?}

Für Personen, die einerseits wirtschaftlich von einem Arbeitgeber (Auftraggeber) abhängig sind, andererseits jedoch zunehmend unternehmerische Merkmale aufweisen, wurde bereits der Begriff „Arbeitskraftunternehmer" kreiert. Der Arbeitende verkauft nicht mehr sein latentes Arbeitsvermögen, sondern handelt (inner- oder überbetrieblich) vorwiegend als Auftragnehmer für Arbeitsleistung. Er organisiert und kontrolliert seine Arbeitskraft weitgehend selbst, um sie dann in den betrieblichen Ablauf einzubringen (z. B. als Verkäufer auf eigenes Risiko Käufer finden). Ein großer Teil der Arbeitskraftunternehmer arbeitet als inner- oder überbetrieblicher Dienstleister und Zulieferer, ohne aber gegen die Marktmacht des Abnehmers (der beschäftigenden Firma) etwas aufbieten zu können. ${ }^{36}$ Werkvertragsnehmer entsprechen diesem Bild sehr gut.

Was bedeutet diese Entwicklung für den heimischen Arbeitsmarkt? Zweifellos weist die Statistik für den Bereich der Selbstständigkeit und neuen Selbstständigkeit eine starke Dynamik auf, wenngleich die Absolutzahlen in Relation zur Gesamtbeschäftigung derzeit noch wenig dramatisch erscheinen. Ob und inwieweit das Modell des standardisierten Berufs und der rechtlich geschützte Status des noch immer dominanten Arbeitnehmers in seiner gesellschaftlichen Leitfunktion für die Vermitt- 
lung zwischen Betrieb, Arbeitsmarkt und Arbeitskraft an Bedeutung verlieren, lässt sich gegenwärtig schwer abschätzen. ${ }^{37}$ Eher unwahrscheinlich erscheint die vollständige Ablöse des unselbstständigen, persönlich und wirtschaftlich abhängigen Arbeitnehmers durch den vorhin skizzierten Typus des Arbeitskraftunternehmers. Abgesehen davon, dass ein derartig radikaler Umbruch gesellschaftlich vermutlich gar nicht erwünscht wäre, halten sich die Vorteile neuer Selbstständigkeit und Selbstständigkeit für alle Beteiligten in Grenzen. Die Möglichkeit der Gewinn bringenden Freisetzung kreativer und innovativer Kräfte, verbunden mit dem angenehmen Gefühl von Eigenständigkeit und persönlicher Verantwortung, wird sich wohl nur einem kleinen, hoch qualifizierten und gefragten Kreis an Personen eröffnen. Das sind jene, die „es sich aussuchen können“. Nicht zu übersehen sind aber auch die Folgen für viele Betroffene abseits der neuen Welt des (Mit-)Unternehmertums: zunehmende Vereinzelung, eine neue Ungleichheit und permanente Selbstausbeutung, dazu hohe Abstiegsrisiken und die Erosion der Solidarität durch Ideologisierung von Erfolgs- und Leistungsnormen. ${ }^{38}$

Auch für Unternehmen relativieren sich mit zunehmender Dauer die auf den ersten Blick erkennbaren (Kosten-)Vorteile. Mit temporären, auftragsbezogenen Verträgen haben Auftrags-/Arbeitnehmer wenig Anreiz, firmenspezifische Kenntnisse zu erwerben, sondern auch anderswo verwertbare. Eine Firma, die zu sehr auf extern-numerische Flexibilität, also auf eine hohe Fluktuation der Arbeitskräfte setzt, wird demgemäß keine firmenspezifischen Fähigkeiten entwickeln können und begrenzt sein in ihrer Möglichkeit, neue Chancen zu erkennen und zu nutzen: Sie wird in Bezug auf neue Ideen an betrieblicher Magersucht leiden. Tichy (2002) unterscheidet in diesem Zusammenhang zwischen negativer und positiver Flexibilität. Negative Flexibilität umfasst die Freiheit von Bindungen durch längerfristige Arbeits-, Liefer- oder Bezugsverträge; sie ermöglicht es, die Firma jederzeit ohne besondere Kosten aufzulösen. Positive Flexibilität hingegen ist die Fähigkeit, neu auftretende Chancen rasch zu ergreifen. Negativ ist die erste Form deshalb, weil sie nur auf die Bewältigung unmittelbarer, kurzfristiger Aufgaben abzielt, aber den Aufbau nachhaltiger Kompetenz verhindert, was in einer (Wirtschafts-)Welt mit komplexitätsbedingten Unsicherheiten und Entwicklungsbrüchen von zentraler Bedeutung wäre. Das ausschließlich auf die Gegenwart fixierte und abgeschlankte Unternehmen beraubt sich so seiner Möglichkeit, auf unvorhergesehene Entwicklungen erfolgreich zu reagieren. Angesichts einer komplexen Welt mit unsicheren Entwicklungsperspektiven müssten die traditionellen Optimierungsstrategien durch Überlebensstrategien ersetzt werden. ${ }^{39}$ Aus unternehmerischer Sicht ist deshalb zu hinterfragen, inwieweit diskontinuierliche Arbeit im Allgemeinen die Qualität der für die Innovations- und Lernfähigkeit von Unternehmen wichti- 
gen Sozialbeziehungen ermöglicht. Der Faktor Vertrauen, der das Engagement und die Kooperationsbereitschaft der Arbeitnehmer innerhalb des Betriebes stärkt, könnte der weiteren Verbreitung derartiger Beschäftigungsverhältnisse Grenzen setzen. Wo individuelle Beschäftigungsunsicherheit wahrgenommen wird, herrscht ein Klima des Misstrauens, das möglicherweise mit nicht kooperativem Verhalten der Beschäftigten einhergeht. Dem betriebswirtschaftlichen Interesse an einer möglichst groBen Flexibilität des Personals in Abhängigkeit von der Auftragslage steht somit das Interesse an einer hohen Leistungsbereitschaft und Produktivität der Arbeitnehmer entgegen. Letzteres setzt das Vertrauen der Beschäftigten voraus und lässt sich am ehesten in stabilen und auf Dauer angelegten Beschäftigungsverhältnissen erreichen. Die maximale Personalflexibilität in einem Betrieb muss deshalb nicht unbedingt gleichbedeutend sein mit der optimalen. ${ }^{40}$

Den Wandel der Unternehmens- und Arbeitsorganisation haben aber nicht nur die Randbelegschaften (,,Outsider"), sondern zunehmend auch die Kernbelegschaften (,Insider") zu spüren bekommen. Eine Vielzahl „neuer" Managementkonzepte wie Lean Production, Business Reengineering, Total Quality Management, Virtuelles Unternehmen usw. wird seit Jahren von emsigen Unternehmensberatern propagiert, angeboten und von vielen Firmen auch angenommen. Dass die Halbwertszeiten dieser Konzepte sich ständig verkürzen, beruht nicht zuletzt auf dem kommerziellen Interesse der Berater am „Veralten“ vorherrschender Problemlösungen. Ziel von Restrukturierungen und Reorganisationen ist meistens die Optimierung der Wertschöpfungskette und damit die Erhöhung des Shareholder Value, das heißt, das ganze Unternehmen, einschließlich seiner externen Partner (Zulieferer, Abnehmer und Distributeure), wird unter Gesichtspunkten der Einsparung von Kosten und Zeit sowie der Prozess- und Produktqualität einer kritischen Überprüfung unterzogen. Für die Stammbelegschaft bedeutet dies, dass das Leitbild des "internen Unternehmertums“ nicht nur für die Führungskräfte, sondern auch für sie selbst verbindlich wird. Der Beschäftigte soll so zum mitdenkenden Unternehmer erzogen werden, dem Rationalisierung nicht mehr angeordnet werden muss, weil er Rationalisierung in Eigenregie betreiben soll. Flachere Hierarchien zielen auf eine Beschleunigung und Optimierung von Arbeitsabläufen ab und delegieren Verantwortung von der Führungsebene auf den „einfachen“ Mitarbeiter. Team- und gruppenarbeitsförmige Organisationsmuster sollen die herkömmliche „Misstrauensorganisation" durch eine "Vertrauensorganisation“ ersetzen. ${ }^{41}$ "Macht, was ihr wollt, aber seid profitabel", könnte man die dahinter stehende Leitidee charakterisieren.

Durch die Hereinnahme des Marktes in die Unternehmen, die sich in der Forderung nach unternehmerischem Denken der Mitarbeiter mani- 
festiert, stehen auch die Kernbelegschaften vor neuen Herausforderungen, denen aber nicht alle gewachsen sind. Zum einen entsteht für die Arbeitnehmer der Zwang zur permanenten (Weiter-)Qualifizierung, zum anderen ist auch eine Intensivierung der Arbeit als Folge der Integration von vorher getrennten Aufgabenbereichen festzustellen. Aufgabenintegration ist ja nicht nur mit dem Abbau von Hierarchiestufen, der Delegation von Verantwortung "nach unten“ und teamartiger Kooperation verbunden, sondern auch mit der Übernahme von Routinetätigkeiten durch hoch qualifizierte und jede Menge unbezahlte Mehrarbeit. ${ }^{42}$ Die vielerorts gestellte Forderung nach „lebenslangem“ Lernen bedeutet in vielen Fällen nichts anderes als die permanente Anpassung des Mitarbeiters an neue Arbeitssituationen und Erfordernisse, die, abgesehen von technologiebedingten Qualifikationserfordernissen, oft einfach dadurch entstehen, dass Aufgaben der Führungsebene delegiert werden und Verantwortung abgeschoben wird. Ein wesentlicher Aspekt der Veränderungen der Arbeitswelt scheint somit auch der Anspruch an die Arbeitnehmerlnnen zu sein, sich unternehmerische Fähigkeiten anzueignen und unternehmerische Aufgaben wahrzunehmen. Mit dieser Forderung sehen sich sowohl atypisch Beschäftigte als auch Dienstnehmerlnnen in Normalarbeitsverhältnissen zunehmend konfrontiert.

\section{Schlussbemerkungen}

Wie bereits in der Einleitung dieser Zusammenfassung dargestellt, bezeichnet der Sammelbegriff „atypische Beschäftigung“ unterschiedliche Formen von Beschäftigungsverhältnissen. Eine genaue und allgemein gültige Beurteilung der zusammengefassten heterogenen Beschäftigungsformen ist aus mehreren Gründen schwierig:

Arbeits- und sozialrechtliche Regelungen differieren je nach Art der Beschäftigung. Teilzeitbeschäftigte sind beispielsweise im Vergleich zu Personen mit freien Dienstverträgen aus arbeits- und sozialrechtlicher Sicht besser gestellt.

Die Motivlagen der betroffenen Personen sind - wie bereits dargestellt - höchst unterschiedlich. Je nach Alter, Bildungsgrad, Geschlecht und aktueller Lebenssituation kann ein und dieselbe Beschäftigungsform zu unterschiedlichen Bewertungen führen, was einschlägige Studien auch zeigen.

Das bereits verwendete Kriterium der Freiwilligkeit ist insofern unbefriedigend und wenig hilfreich, als eine Grenzziehung zwischen Freiwilligkeit und Zwang letztlich nicht möglich ist. Da Erwerbsarbeit, wie schon der Begriff ausdrückt, in der Regel die Grundlage bildet, um einen (existenzsichernden) Erwerb zu erzielen, relativiert sich die Freiwilligkeit nicht nur atypisch Beschäftigter. Die Klassifizierung der Wahl von Beschäfti- 
gungsformen in „freiwillig“ und „unfreiwillig“ bzw. die Interpretation von Umfragen bietet deshalb nur eine ungenaue, nicht klar definierte Basis zur Beurteilung atypischer Beschäftigungsverhältnisse ${ }^{43}$.

Gerade im Bereich atypischer Beschäftigung spielt der Zeithorizont eine wesentliche Rolle. Aufgrund ihres im Vergleich zur Normalbeschäftigung prekären Charakters bieten atypische Beschäftigungsformen zwar kurz- und mittelfristig durchaus Möglichkeiten einer von Arbeitnehmerlnnen erwünschten Flexibilität, werden aber mit zunehmender Dauer unattraktiv. Langfristig, so könnte man es auf den Punkt brịgen, muss man sich atypische Beschäftigung leisten können.

Maßstab für die arbeits- und sozialrechtliche Beurteilung von atypischer Beschäftigung sollte nach wie vor das klassische Normalarbeitsverhältnis - mit all seinen Pflichten, aber auch Rechten und Ansprüchen für den Arbeitnehmer - sein. Dies umso mehr, als einige Formen atypischer Beschäftigung (Werk- und freie Dienstverträge) von immer mehr Unternehmen dazu missbraucht werden, das System kollektiver Mindestlöhne und arbeitsrechtlicher Mindeststandards zu umgehen. Sachlich zu rechtfertigen ist die krasse arbeitsrechtliche Benachteiligung von freien Dienstnehmern ebenso wenig wie deren Ausschluss aus der Arbeitslosenversicherung. Der freie Dienstnehmer in seiner jetzigen Form kann aus Sicht einer Arbeitnehmerinteressenvertretung wohl nur als Fehlkonstrukt bezeichnet werden. Die juristische Herausforderung besteht darin, den Begriff des Arbeitnehmers umfassender zu definieren und vom Kriterium der persönlichen Abhängigkeit so weit zu lösen, dass auch freie Dienstnehmer in den Genuss arbeitsrechtlicher Ansprüche kommen.

Weitaus komplexer erweist sich der Bereich der neuen Selbstständigkeit. Die Forderung nach einer eindeutigen Abgrenzung zwischen Unselbstständigkeit und Selbstständigkeit liegt zwar nahe, ist aber sowohl theoretisch als auch praktisch schwer zu bewerkstelligen. Gerade hier zeigt sich, dass konzeptionelle Überlegungen bedeutend leichter $\mathrm{zu}$ formulieren als in gesellschaftliche Praxis umzusetzen sind. $\mathrm{Zu}$ berücksichtigen ist außerdem, inwieweit legislative Korrekturen und Ergänzungen geeignet sind, Ziele zu erreichen, oder ob sie sich letztlich nicht doch als kontraproduktiv herausstellen, indem sie unerwünschte Entwicklungen auslösen oder beschleunigen. Gerade das Beispiel des freien Dienstvertrages zeigt, wie schwer einmal geöffnete Schleusen wieder zu schließen sind. Vor diesem Hintergrund scheint eine arbeitsund sozialrechtliche Besserstellung von neuen Selbstständigen problematisch. Selbst wenn es gelänge, den Arbeitnehmerbegriff derart weit zu fassen, dass auch Werkvertragsnehmer in den Geltungsbereich des Arbeitsrechts fielen, müsste man in einem zweiten Schritt die Auftraggeber zur Abgabe von Sozialversicherungsbeiträgen für „ihre“ Auftragneh- 
mer verpflichten. ${ }^{44}$ Andernfalls würde man eine Form von Arbeitnehmer schaffen, die zwar arbeitsrechtlichen Schutz genießt, aber hinsichtlich Sozialabgaben eine wesentlich günstigere Variante für den Auftraggeber darstellt. Ähnlich dem freien Dienstnehmer hätte man dann eine für Unternehmen praktikable und kostengünstige Alternative zum arbeitsund sozialrechtlich voll eingebetteten normalen Dienstnehmer geschaffen. Nicht dass Werkvertragsnehmer diese Funktion nicht jetzt schon hätten, aber eine verstärkte arbeits- und sozialrechtliche Einbindung hätte zur Folge, eine grundsätzlich problematische Vertragsform salonfähig zu machen. Es wäre paradox, wenn ausgerechnet die Arbeitnehmervertretungen dem Werkvertrag die Salonfähigkeit attestieren würden. Ein Zitrone bleibt sauer, auch wenn man sie zuckert. Wesentlich, wenn auch nicht sonderlich originell und immer hilfreich, wäre in diesem Zusammenhang verstärkte Information über die Risiken von (neuer) Selbstständigkeit.

Die allgemeine Herausforderung für die Arbeitnehmerinteressenvertretungen angesichts der Zunahme heterogener Beschäftigungs- und Vertragsformen besteht darin, die Grenze zwischen individuell erwünschter, sozialpolitisch vertretbarer Flexibilität und Missbrauch aus Sicht der ArbeitnehmerInnen zu definieren. In Anbetracht zersplitterter Interessen, unterschiedlicher Motiv- und Lebenslagen der Betroffenen ist dies kein einfaches Unterfangen und kommt teilweise einer Quadratur des Kreises gleich.

Aus arbeitsmarktpolitischer Perspektive könnten atypische Beschäftigungsverhältnisse grundsätzlich eine für ArbeitnehmerInnen hilfreiche Funktion haben, soferne sie die Brückenfunktion zwischen Ausbildung oder Arbeitslosigkeit auf der einen Seite und arbeits- und sozialrechtlich abgesicherter (Vollzeit-)Tätigkeit auf der anderen Seite erfüllen. Der Kontakt mit der Arbeitswelt im Allgemeinen bzw. mit Unternehmen im Besonderen beispielsweise über Werkverträge oder befristete Verträge bietet allen Beteiligten die Möglichkeit des gegenseitigen Kennenlernens. Wichtig aus Arbeitnehmerlnnensicht ist freilich die Aussicht auf ein Ende derartiger Probephasen auf Basis atypischer, teilweise prekärer Beschäftigungsformen. Sollte diese Perspektive nicht gegeben sein, tragen atypische Beschäftigungsverhältnisse dazu bei, eine Struktur von Insidern und Outsidern auf dem Arbeitmarkt zu schaffen und bestehende Ungleichheiten zu verstärken.

Die veränderte Struktur des Arbeitsmarktes muss für die Beurteilung und Darstellung der Situation auf dem Arbeitsmarkt Konsequenzen haben. Angesichts der in diesem Beitrag skizzierten Entwicklung zielt die Kritik, der österreichische Arbeitsmarkt sei unflexibel und bedürfe deshalb einer „Deregulierung“ und „Flexibilisierung“, ins Leere. ${ }^{45}$ Der heimische Arbeitsmarkt weist in der Realität ein hohes Maß an Beweglichkeit 
auf, mit all den Chancen, aber auch negativen Folgen für die Betroffenen. Der oftmals unterstellte Zusammenhang zwischen hoher Flexibilität und geringer Arbeitslosigkeit scheint indes keine empirische Bestätigung zu finden. Schließlich zeigen internationale Vergleiche, dass der Zusammenhang zwischen Flexibilitätsgraden des Arbeitsmarktes und dem Beschäftigungsniveau aus empirischer Perspektive weit weniger eindeutig ist, als aufgrund spezifischer (neoklassischer) Annahmen über die Funktionsweise von Arbeitsmärkten zu erwarten wäre. Deregulierungen beeinflussen demnach eher Dynamik und Zusammensetzung der Arbeitslosigkeit als deren Niveau ${ }^{46}$ Insgesamt wird dem quantitativen Aspekt, nicht nur in der Arbeitsmarkt- und Beschäftigungspolitik, eine zu große Bedeutung beigemessen. Die (vermeintlichen) Siege an der beschäftigungspolitischen Zahlenfront in Form von Rekorderwerbsbeteiligungen dürfen nicht darüber hinwegtäuschen, dass sich das Hinterland qualitativ befriedigender, existenzsichernder Arbeitsplätze zum Teil in einem schlechten Zustand befindet. Abseits juristischer Flickschusterei ist deshalb dem qualitativen Aspekt von Arbeit gerade in Zeiten internationaler Erwerbsbeteiligungswettbewerbe verstärkt Aufmerksamkeit zu schenken. Der Frage, inwieweit die Fixierung auf Ziele wie Vollbeschäftigung und erhöhte Erwerbsbeteiligung eine ausreichende Berücksichtigung dieses Aspekts zulässt, werden auch die Interessenvertretungen der Arbeitnehmer auf Dauer nicht ausweichen können.

Last but not least sei (wieder einmal) daran erinnert, dass die beste Angebotspolitik kurzfristig wenig bewirkt, wenn die Nachfrageseite wirtschaftspolitisch vernachlässigt wird. Eine noch so große Flexibilität auf Arbeitsmärkten wird die negativen Folgen restriktiver, prozyklischer Budgetpolitik und einer ausschließlich auf Inflationsbekämpfung ausgerichteten Geldpolitik auf europäischer Ebene nur in geringem Ausmaß kompensieren können.

\section{Anmerkungen}

1 So ist z. B. Teilzeitarbeit aus Sicht der Männer als nicht der Norm entsprechend, also atypisch, zu qualifizieren, während sie für viele Frauen der Realität und somit auch „Normalität" ihrer Erwerbstätigkeit entspricht. Die Heterogenität der unter dem Sammelbegriff „atypische Beschäftigung“ zusammengefassten Vertragsarten erschwert außerdem eine generelle Beurteilung und verstellt mitunter den Blick auf spezifische Chancen und Risiken bestimmter Arbeitsverhältnisse.

2 Die Angaben beziehen sich auf Beschäftigungsverhältnisse und nicht auf Beschäftigte.

3 Vgl. Kirisits (2002) für die Steiermark, Holzinger et al. (2000) für Gesamtösterreich sowie Arbeiterkammer Tirol ( 2002) für Tirol.

${ }^{4} \mathrm{Vgl}$. Regionalstatistiken der AK Steiermark.

5 Unselbstständig Beschäftigte mit einer wöchentlichen Arbeitszeit von mindestens 12 und maximal 35 Stunden, ohne Präsenz- und Zivildiener, Karenzurlauberinnen (Lebensunterhaltskonzept). Für 2001 handelt es sich um vorläufige Ergebnisse. 
6 Geringfügig beschäftigt sind Personen, deren Entgelt nicht über die Geringfügigkeitsgrenze von 309,38 Euro (4.149,30 Schilling) pro Kalendermonat hinausgeht. Geringfügig Beschäftigte werden vom Hauptverband der Sozialversicherungsträger erst seit Ende 1994 erfasst, da die Betriebe seit damals zur Meldung verpflichtet sind.

7 Die Wirtschaftsklasse „Unternehmensbezogene Dienstleistungen“ enthält so unterschiedliche Sparten wie Rechts-, Steuer- und Unternehmensberatung, Architektur- und Ingenieurbüros, Labors und Versuchsanstalten, Werbewesen und Reinigungsgewerbe. Vor allem das Reinigungsgewerbe dürfte im Zusammenhang mit geringfügiger Beschäftigung von zentraler Bedeutung sein.

8 Auswertung des Hauptverbandes der österreichischen Sozialversicherungsträger (1. Juli 2001).

9 Von einem befristeten Arbeitsverhältnis wird gesprochen, wenn eine Arbeitskraft nur für einen bestimmten, vorher festgelegten Zeitraum beschäftigt wird oder wenn ein Dienstvertrag für eine spezifische Aufgabe mit begrenzter Dauer vorliegt. Die Anzahl der befristeten Arbeitsverhältnisse wurde erstmals in der Arbeitskräfteerhebung der EU im Jahr 1995 erfasst.

${ }^{10} \mathrm{Vgl}$. Statistik Austria, Arbeitskräfteerhebung März 2001.

${ }^{11}$ Im März 1988 wurde erstmals ein umfassendes Arbeitskräfteüberlassungsgesetz (AÜG) beschlossen. Durch dieses Gesetz wurden Leiharbeitsfirmen (Überlasser) unter anderem verpflichtet, laufende Aufzeichnungen über die Überlassungen von Arbeitskräften zu führen und diese per Stichtag 31 . Juli an die zuständigen Landesgeschäftsstellen des Arbeitsmarktservice (AMS) zu übermitteln.

${ }^{12}$ Freie Dienstnehmer: Dienstnehmer, die gemäß $\S 4$ Abs. 4 ASVG versichert sind.

${ }^{13}$ Werkvertragsnehmer, die gemäß § 2 Abs. 1 Z 4 GSVG pflichtversichert sind. Die Zahlen enthalten keine Wirtschaftstreuhänder, Dentisten, Journalisten und Tierärzte. Die tatsächliche Zahl der neuen Selbstständigen ist höher anzusetzen, da im Bereich der neuen Selbstständigkeit Personen von der Sozialversicherung erst dann erfasst werden, wenn ihr Einkommen zumindest den 12fachen Betrag der Geringfügigkeitsgrenze (derzeit € 309,38) übersteigt.

${ }^{14}$ Die angeführten Beispiele gelten natürlich nur mit Einschränkungen. So sind auch Werkvertragsnehmer ab einer bestimmten Einkommensgrenze sozialversicherungspflichtig, Beamte müssen nicht unbedingt Vollzeitbeschäftigte sein.

${ }^{15} \mathrm{Vgl}$. Kaupa/Steiner (2002) 126. Abgesehen von den Problemen, die "Intensität“ der Prekarisierung im Sinne einer Messung vorzunehmen (um eine Abgrenzung zu nicht prekären Arbeitsverhältnissen vorzunehmen), impliziert die Klassifizierung ein (anzustrebendes) Standardmodell von Beschäftigung, das durch Vollzeitlichkeit, Kontinuität, geregeltes Einkommen sowie arbeits- und sozialrechtliche Absicherung gekennzeichnet ist. Die Tatsache, dass (Be-)Wertungen vom sozio-kulturellen Kontext abhängig sind, schränkt den Geltungsbereich derartiger Klassifizierungen zwar ein, macht sie aber als Grundlage für Handlungsempfehlungen auf nationaler Ebene nicht weniger wertvoll. Letztlich stehen hinter jeder Interpretation des Prekaritätsbegriffs Wertvorstellungen, über deren Richtigkeit sich wissenschaftlich nicht abschließend urteilen lässt. In einem Kulturkreis wie den USA, wo Selbstständigkeit und Unternehmertum einen weitaus größeren Stellenwert besitzen als in Österreich, wird unter prekärer Beschäftigung vermutlich etwas anderes verstanden als hierzulande. Vgl. dazu auch die Beiträge zum Thema „Arbeit im Vergleich der Kulturen“, in: Kocka, Jürgen; Offe, Claus (Hrsg.), Geschichte und Zukunft der Arbeit (Frankfurt/Main 2000).

${ }^{16} \mathrm{Vgl}$. Angerler/Kral-Bast (1998) 40 und Juffinger (2000) 22.

${ }^{17}$ Vgl. Fink/Riesenfelder/Tálos (2001) 120.

${ }^{18}$ Ebd. 153. 
${ }^{19} \mathrm{Vgl}$. die (falsche) Darstellung der Studienergebnisse von Fink/RiesenfelderfTálos durch Wirtschats- und Arbeitsminister Martin Bartenstein. der die Studie dahingehend interpretierte, dass die meisten Menschen, die eine atypische Beschäftigung ausüben, mit ihrer beruflichen Situation zufrieden seien („Der Standard", 15.12.2001).

${ }^{20}$ Auf die grundlegende Frage, inwieweit Menschen überhaupt freiwillig arbeiten, soll hier nicht eingegangen werden. Die Unterscheidung zwischen Freiwilligkeit und Unfreiwilligheit bezieht sich im Rahmen dieser Arbeit auf den Wunsch von Personen, in Vollzeit und unbefristet zu ai beiten, und die Möglichkeit, dies auch zu verwirkt ichen.

${ }^{21} \mathrm{Vgl}$. Fink/Riesenfelder/Tàlos (2001) 116.

${ }^{22}$ Ergebnisse der Arbeitskräfteerhebung 1998, in: Statistische Nachrichten 2 (2000).

${ }^{23} \mathrm{Vgl}$. Schwarz/Lös chnigg (2000) $247 \mathrm{ff}$.

${ }^{24} \mathrm{Vgl}$. Mühlberger (1998a) 49.

${ }^{25}$ Juffinger (2000) hat verschiedene Leiharbeitertypen auf Basis ihrer Untersuchung herausgearbeilet. An 315 Leiharbeiterinnen wurde ein Fragebogen verschickt, und mit 46

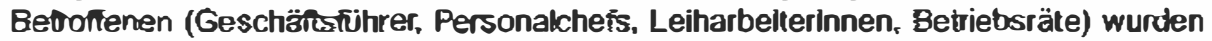
Interviews geführt.

${ }^{26} \mathrm{Vgl}$. Mosberger/Steiner (2002) 70f. Hinsichtlich der Verteilung auf die beiden Kategorien geben die Autorinnen für die „aus der Not geborenen" Selbstständigen einen Anteil von etwa 50 Prozent an. Zweifellos liefern diese Aussagen Aufschlüsse über die Motivlagen neuer Selbstständiger; deren Verteilung muss jedoch angesichts der geringen Anzahl befragter Personen $(n=30)$ mit Vorsicht interpretiert werden.

27 Vgl. Reindl (2000) 425.

${ }^{28} \mathrm{Vgl}$. Schwaiz/Löschnigg (2000) $141 \mathrm{f}$.

${ }^{29}$ Hinsichtlich des Begriffs der "wirtschaftlichen Abhängigkeit" eines Arbeitnehmers ist die Judikatur jedoch uneinheitlich. Einige Entscheidungen rücken die Lohnabhängigkeit und damit das Entgelt als Existenzgrundlage in den Vordergrund, während die Rechtsprechung in anderen Fällen das fehlende Eigentum an Produktionsmitteln bzw. die fehlende Verfügungsgewalt über dieselben als wirtschaftiche Abhängigkeit interpretiert. (Vgl. Schwarz/Löschnigg (2000) 142.

${ }^{30} \mathrm{Vg}$. Tomandl (1999) $295 f$.

${ }^{31}$ Vgl. Grewer/Reindl (2000) 3.

32 Vgl. Reindl (2000) 415.

${ }^{33}$ Der Einzelunternehmer gilt als natürliche Person, ist für die Aufbringung des Kapitals alleine zuständig, trägt das volle Risiko für etwaige Verluste und haftet mit seinem Privatvermögen. Die Daten der Wirtschaftskammer beinhalten nur Tätigkeiten, die der Gewerbeordnung unterliegen und somit einer Gewerbeberechtigung (Gewerbeschein) bedürfen. Wer eine gewerbliche Tätigkeit ausübt, ist automatisch Mitglied der Wirtschaftskammer.

${ }^{34} \mathrm{Vgl}$. Schmölzer/Karner (2001) $1 \mathrm{ff}$.

${ }^{35}$ Wanzenbäck, Das österreichische Gründungsgeschehen - Die Zahlen, Daten, Fakten. Kurzbericht abrufbar unter http://www.travelguide.at/businessguide/gs_zahlen.html.

${ }^{36} \mathrm{Vgl}$. Voß/Pongratz (1998) 139ff.

${ }^{37} \mathrm{Vgl}$. JurczykNoß (1999) 10.

${ }^{38} \mathrm{Vgl.} \mathrm{Haak} /$ Schmid (2001) 172.

$39 \mathrm{Vgl}$. Tichy (2002) $175 \mathrm{ff}$.

${ }^{40} \mathrm{Vgl}$. SeiferUPawlonsky (1998) 599.

${ }^{41}$ Vgl. Müller-Jentsch (1998) $576 f$.

42 Vgl. Heschi/Kirisits (2000) 44.

${ }^{43}$ Auch die hohe Teilzeitquote bei Frauen ist vor diesem Hintergrund zu sehen, da das mangelhafte Angebot an Kinderbetreuungseinrichtungen in Osterreich Frauen oft in dieses Arbeitsmark tsegment zwingt. 
${ }^{44}$ Was praktisch nicht durchführbar wäre, da die Auftraggeber diese in Form verminderter Honorarzahlungen de facto auf die Auftragnehmer abwälzen könnten.

${ }^{45}$ Diese Diskussion wird allerdings schon deshalb immer wieder Konjunktur haben, weil sie mit schwammigen Begriffen operiert. Man wird schwer Einigkeit darüber erzielen können, ab wann ein Arbeitsmarkt "flexibel" ist. Nichtsdestoweniger ist es Aufgabe der Arbeitnehmerinteressenvertretungen, auch auf die sozialen Kosten beruflicher Flexibilität hinzuweisen. Der Begriff „Deregulierung“ wird nicht nur im arbeitsmarktpolitischen Zusammenhang falsch verwendet. Er suggeriert einen von Regulierungen und Bindungen befreiten Zustand, obwohl Wettbewerb nicht im regulierungsfreien Umfeld stattfindet, sondern oft nur auf Basis eines noch komplizierteren Regelwerks möglich ist (siehe die Bereiche Energie und Telekommunikation). Korrekterweise müsste man von Re-Regulierungen sprechen.

${ }^{46} \mathrm{Vgl}$. Keller/Seifert (2002) 92.

\section{Literatur}

Angerler, Eva; Kral-Bast, Claudia, Typische Atypische. Flexible Arbeit - Teil ॥ (= Publikation der Gewerkschaft der Privatangestellten, Wien 1998).

Arbeiterkammer Tirol (Hrsg.), Die Lage der Arbeitnehmer in Tirol 2002 (Innsbruck 2002).

Fink, Marcel; Riesenfelder, Andreas; Tálos, Emmerich, Endbericht Atypische Beschäftigungsverhältnisse. Geringfügige Beschäftigung und freie Dienstnehmerlnnen (Wien 2001).

Grewer, Hans Günter; Reindl, Josef, Scheinselbstständige im Saarland (= Teil 2 einer Studie über Tendenzen zur Auflösung des Normalarbeitsverhältnisses durch die Zunahme ungesicherter Beschäftigungsverhältnisse, Saarbrücken 2000).

Haak, Carroll; Schmid, Günther, Arbeitsmärkte für Künstler und Publizisten: Modelle der künftigen Arbeitswelt?, in: Leviathan 2 (2001) 156-178.

Heschl, Franz; Kirisits, Marcel, „Es weht ein rauerer Wind!“. Aspekte eines Stimmungsbildes zu den Auswirkungen von EU-Mitgliedschaft, Ostöffnung und Globalisierung in steirischen Industrieunternehmen (= Publikation der AK Steiermark, Graz 2000).

Hoffmann, Edeltraut; Walwei, Ulrich, Normalarbeitsverhältnis: ein Auslaufmodell? Überlegungen zu einem Erklärungsmodell für den Wandel der Beschäftigungsformen, in: Mitteilungen aus der Arbeitsmarkt- und Berufsforschung 3 (1998) 409-425.

Höglinger, Andrea, „Schöne neue Arbeitswelt?“ Endbericht zum Forschungsprojekt „Atypische Beschäftigungsverhältnisse" des Zentrums für soziale Innovation (Wien 1996)

Holzinger, Elisabeth; Federer, Maria Louisa; Hovorka-Prentner, Sylvia; Woschnak, Gerhard; Schmied, Gabriele; Wach, Iris, Atypische Beschäftigungsverhältnisse. Entwicklungstrends und Handlungsoptionen (Wien 2000).

Juffinger, Sabine, Leiharbeit in Tirol. Chance oder Ausbeutung? (Innsbruck 2000).

Jurczyk, Karin; Voß, G. Günter, Entgrenzte Arbeitszeit - Reflexive Alltagszeit. Die Zeiten des Arbeitskraftunternehmers (München/Gießen 1999).

Kaupa, Isabella; Steiner, Karin, Atypische Beschäftigung: Notlösung, Übergangsstadium oder freie Wahl?, in: Eichmann, Hubert; Kaupa, Isabella; Steiner, Karin (Hrsg.), Game over? Neue Selbstständigkeit und New Economy nach dem Hype (Wien 2002) 121-139.

Keller, Berndt; Seifert, Hartmut, Flexicurity - Wie lassen sich Flexibilität und soziale Sicherheit vereinbaren?, in: Mitteilungen aus der Arbeitsmarkt- und Berufsforschung 1 (2002) 90-106.

Kirisits, Marcel, Schein und Sein der neuen Arbeitswelt. Atypische Beschäftigung in der Steiermark (= Publikation der AK Steiermark, Graz 2002). 
Mosberger, Brigitte; Steiner, Karin, Unternehmerisches Agieren oder flexibles Reagieren. Situation und Erwerbsrealität Neuer Selbstständiger in Österreich (= AMS Report 32, Wien 2002).

Mühlberger, Ulrike, Atypische Beschäftigung in Österreich. Sozial- und arbeitsmarktpolitische Implikationen atypischer Beschäftigungsverhältnisse (Wien 1998).

Müller-Jentsch, Walther, Der Wandel der Unternehmens- und Arbeitsorganisation und seine Auswirkungen auf die Interessenbeziehungen zwischen Arbeitgebern und Arbeitnehmern, in: Mitteilungen aus der Arbeitsmarkt- und Berufsforschung 3 (1998) 575-584.

Reindl, Josef, Scheinselbstständigkeit. Ein deutsches Phänomen und ein verkorkster Diskurs, in: Leviathan 4 (2000) 413-433.

Tichy, Gunther, Die Herausforderung der Wissensgesellschaft. Trügerische versus erfolgversprechende Strategien für die e-society, in: Wirtschaft und Gesellschaft 2 (2002) 171-184.

Tomandl, Theodor, Reicht der Zuschnitt unseres Arbeitsrechts zur Bewältigung der Zukunftsaufgaben?, in: Pichler, Johannes W. (Hrsg.), Die "Neue“ Arbeit. Die rechtspolitischen Herausforderungen (Wien 1999) 293-308.

Schmölzer, Gertrude; Karner, Beatrix, Unternehmensneugründungen in der Steiermark 2000 (Graz 2001).

Schwarz, Walter; Löschnigg, Günther, Arbeitsrecht (Wien 2000, 8. Auflage.)

Seifert, Matthias; Pawlowsky, Peter, Innerbetriebliches Vertrauen als Verbreitungsgrenze atypischer Beschäftigungsformen, in: Mitteilungen aus der Arbeitsmarkt- und Berufsforschung (MittAB) 3 (1998) 599-611.

Voß, G. Günter; Pongratz, Hans J., Der Arbeitskraftunternehmer. Eine neue Grundform der Ware Arbeitskraft?, in: Kölner Zeitschrift für Soziologie und Sozialpsychologie 1 (1998) 131-158.

\section{Zusammenfassung}

Seit mehr als zwei Jahrzehnten ist auf dem österreichischen Arbeitsmarkt eine Zunahme von Beschäftigungsverhältnissen festzustellen, die vom so genannten Normalarbeitsverhältnis, welches durch abhängige, vollzeitliche, kontinuierliche und auf geregelter Arbeitszeit und geregeltem Einkommen basierende Tätigkeit charakterisiert wird, abweichen. Alle Beschäftigungsverhältnisse, die dieser Norm nicht entsprechen, werden in der Literatur unter dem Sammelbegriff „atypische Beschäftigung“ zusammengefasst. Darunter fallen so unterschiedliche Ausprägungen wie Teilzeitarbeit, geringfügige Beschäftigung, befristete Beschäftigung, Leiharbeit und (Schein-)Selbstständigkeit in Form von Werkund freien Dienstverträgen. Die unterschiedliche arbeits- und sozialrechtliche Ausgestaltung dieser Beschäftigungsformen sowie heterogene Motiv- und Lebenslagen betroffener Personen machen eine generelle Beurteilung aus Sicht von Arbeitnehmerinteressenvertretungen schwierig. Vor allem die zunehmende Verbreitung neuer (Schein-)Selbstständigkeit entzieht sich dem tradierten Unternehmer-Arbeitnehmer-Schema und verschleiert nach wie vor bestehende Mechanismen von Machtausübung und Ausbeutung sowie die damit verbundene Externalisierung bestimmter Unternehmerrisiken. Die veränderte Struktur des Arbeitsmarktes wirft schließlich Fragen nach dem Sinn einer im Wesentlichen an quantitativen Zielen orientierten Arbeitsmarkt- und Beschäftigungspolitik auf. 